\title{
HYPERASYMPTOTIC SOLUTIONS OF SECOND-ORDER LINEAR DIFFERENTIAL EQUATIONS II
}

\author{
A. B. Olde Daalhuis
}

\begin{abstract}
In the preceding paper, hyperasymptotic expansions were obtained for the solutions of homogeneous linear differential equations of the second order in the neighborhood of an irregular singularity of rank one. In the present paper, we derive alternative forms of these expansions that are sometimes advantageous.
\end{abstract}

\section{Introduction and summary}

In the preceding paper [1], hereinafter referred to as $\mathbf{I}$, it was shown how to re-expand the asymptotic solutions of the homogeneous linear differential equation

$$
\frac{d^{2} w}{d z^{2}}+f(z) \frac{d w}{d z}+g(z) w=0
$$

in the neighborhood of an irregular singularity of rank one in very accurate series of repeated integrals of the generalized exponential integral (or incomplete Gamma function). The purpose of the present paper is to derive other expansions of this type that have some advantages, as well as disadvantages, over the expansions of $\mathbf{I}$.

We shall assume that the reader is familiar with the main results in $\mathbf{I}$, and we shall use the same notation and adopt the same conditions as in $\S \S 1,2$, and 3 of this reference.

The paper is arranged as follows. In $\S 2$ we give some new definitions and properties pertaining to the "hyperterminants" introduced in I, $\S 2$. The main results appear in the form of three theorems that are stated in $\S 3$ and proved in $\S \S 4-6$. In $\S 7$ a comparison is made of the hyperasymptotic expansions with those of $\mathbf{I}$. The concluding section, $\S 8$, supplies a numerical example.

\section{Auxiliary functions}

We define

$$
\tilde{G}_{\omega}^{(0)}(z ; N)=1, \quad \tilde{G}_{\omega}^{(l)}(z ; N)=G_{\omega}^{(l)}(z ; N, \ldots, N, N+1), \quad l=1,2,3, \ldots,
$$

where $z$ is a real or complex variable and $N$ is an arbitrary positive integer. Thus, from $\mathbf{I}(2.2)$ we have

$$
\tilde{G}_{\omega}^{(l)}(z ; N)=\int_{0}^{\infty} \cdots \int_{0}^{\infty} \frac{e^{-t-t_{1}-\cdots-t_{l-1}} t^{N+\omega_{1}} t_{1}^{N-\omega} \ldots t_{l-1}^{N+(-)^{l-1} \omega}}{(t+z)\left(t_{1}+t\right)\left(t_{2}+t_{1}\right) \ldots\left(t_{l-1}+t_{l-2}\right)} d t_{l-1} \ldots d t_{1} d t
$$

Received June 2, 1994, revised November 3, 1994.

1991 Mathematics Subject Classification. Primary, 34E05; Secondary, 33C10.

Key words and phrases: asymptotic expansions, Bessel functions, connection formulas, differential equations, hyperasymptotics, irregular singularity, remainder term. 
valid when $l \geqslant 1,|\mathrm{ph} z|<\pi$, and $N>|\Re \omega|-1$. As in the case of the functions $G_{\omega}^{(l)}$, the only singularities of the $\tilde{G}_{\omega}^{(l)}$ in the $z$-plane are branch-points, or poles, at $z=0$ and $\infty$. Outside the sector $|\mathrm{ph} z|<\pi$, these functions are defined by analytic continuation.

From $\mathbf{I}(2.9)$ we derive the connection formula

$$
\tilde{G}_{\omega}^{(l)}\left(z e^{-2 \pi i} ; N\right)-\tilde{G}_{\omega}^{(l)}(z ; N)=2 \pi i e^{-(N+\omega) \pi i} e^{z} z^{N+\omega} \tilde{G}_{-\omega}^{(l-1)}\left(z e^{-\pi i} ; N\right), \quad l \geqslant 1 .
$$

Next, if we substitute into the integral

$$
\int_{0}^{\infty} \cdots \int_{0}^{\infty} \frac{e^{-t-t_{1}-\cdots-t_{2 l-1}} t^{N+\omega} t_{1}^{N-\omega} \ldots t_{2 l-2}^{N+\omega} t_{2 l-1}^{N-\omega}}{(t+z)\left(t_{1}+t\right)\left(t_{2}+t_{1}\right) \ldots\left(t_{2 l-1}+t_{2 l-2}\right)\left(e^{-\pi i} z+t_{2 l-1}\right)} d t_{2 l-1} \ldots d t_{1} d t
$$

by means of the identity

$$
(t+z)-\left(t_{1}+t\right)+\left(t_{2}+t_{1}\right)-\cdots-\left(t_{2 l-1}+t_{2 l-2}\right)+\left(e^{-\pi i} z+t_{2 l-1}\right)=0,
$$

we obtain the convolution formula

$$
\sum_{j=0}^{2 l}(-)^{j} \tilde{G}_{\omega}^{(j)}(z ; N) \tilde{G}_{-\omega}^{(2 l-j)}\left(z e^{-\pi i} ; N\right)=0
$$

valid when $l$ is any positive integer.

We shall also need the "constants" defined by

$$
g_{\omega}^{(1)}(M ; k)=\int_{0}^{\infty} e^{-t} t^{M+k-1+\omega} d t
$$

and

$$
\begin{aligned}
& g_{\omega}^{(l)}(M ; k)= \\
& \quad \int_{0}^{\infty} \cdots \int_{0}^{\infty} \frac{e^{-t-t_{1}-\cdots-t_{l-1}} t^{M+\omega} t_{1}^{M-\omega} \ldots t_{l-2}^{M+(-)^{l-2} \omega} t_{l-1}^{M+k-1+(-)^{l-1} \omega}}{\left(t_{1}+t\right)\left(t_{2}+t_{1}\right) \ldots\left(t_{l-1}+t_{l-2}\right)} d t_{l-1} \ldots d t_{1} d t,
\end{aligned}
$$

for $l \geqslant 2$. For $l=1,2$ we have

$$
\begin{aligned}
& g_{\omega}^{(1)}(M ; k)=\Gamma(M+k+\omega) \\
& g_{\omega}^{(2)}(M ; k)=\frac{\Gamma(M+1+\omega) \Gamma(M+k-\omega)}{2 M+k},
\end{aligned}
$$

but closed expansions have not been obtained so far in the cases $l=3,4, \ldots$.

Next, we introduce the functions

$$
\begin{aligned}
P_{\omega}\left(z ; N ; K_{1}, K_{2}\right) & =\sum_{j=0}^{\infty}(-)^{j N} \frac{K_{1}^{j} K_{2}^{j}}{z^{2 j}} \tilde{G}_{\omega}^{(2 j)}(z ; N) \\
Q_{\omega}\left(z ; N ; K_{1}, K_{2}\right) & =\sum_{j=0}^{\infty}(-)^{(j+1) N} \frac{K_{1}^{j+1} K_{2}^{j}}{z^{(2 j+1) N}} \tilde{G}_{\omega}^{(2 j+1)}(z ; N),
\end{aligned}
$$

which we shall call the hyperconnection functions. If we take $N=|z|+\mathcal{O}(1)$, then with the aid of I, Lemma 2.1, we see that the sums in (2.7) converge for $|\mathrm{ph} z| \leqslant \pi$ and 
all sufficiently large $|z|$. Furthermore, if we truncate these infinite sums after $l$ terms, then the remainders are of the same order as the first neglected term; thus

$$
\begin{aligned}
\sum_{j=l}^{\infty}(-)^{j N} \frac{K_{1}^{j} K_{2}^{j}}{z^{2 j N}} \tilde{G}_{\omega}^{(2 j)}(z ; N) & =\tilde{G}_{\omega}^{(2 l)}(z ; N) \mathcal{O}\left(z^{-2 l N}\right) \\
& =\mathcal{O}\left(e^{-2 l|z|} z^{\frac{1}{2}-l}\right), \\
\sum_{j=l}^{\infty}(-)^{(j+1) N} \frac{K_{1}^{j+1} K_{2}^{j}}{z^{(2 j+1) N}} \tilde{G}_{\omega}^{(2 j+1)}(z ; N) & =\tilde{G}_{\omega}^{(2 l+1)}(z ; N) \mathcal{O}\left(z^{-(2 l+1) N}\right) \\
& =\mathcal{O}\left(e^{-(2 l+1)|z|} z^{-l+\Re \omega}\right),
\end{aligned}
$$

as $z \rightarrow \infty$ in $|\mathrm{ph} z| \leqslant \pi$.

From the connection formula (2.3) we obtain

$$
\begin{aligned}
& P_{\omega}\left(z e^{-2 \pi i} ; N ; K_{1}, K_{2}\right)-P_{\omega}\left(z ; N ; K_{1}, K_{2}\right) \\
& \quad=2 \pi i K_{1} e^{-\omega \pi i} e^{z} z^{\omega} Q_{-\omega}\left(z e^{-\pi i} ; N ; K_{2}, K_{1}\right), \\
& Q_{\omega}\left(z e^{-2 \pi i} ; N ; K_{1}, K_{2}\right)-Q_{\omega}\left(z ; N ; K_{1}, K_{2}\right) \\
& \quad=2 \pi i K_{1} e^{-\omega \pi i} e^{z} z^{\omega} P_{-\omega}\left(z e^{-\pi i} ; N ; K_{2}, K_{1}\right) .
\end{aligned}
$$

Also, from (2.7), we have

$$
\begin{aligned}
& P_{-\omega}\left(z e^{-\pi i} ; N ; K_{2}, K_{1}\right) P_{\omega}\left(z ; N ; K_{1}, K_{2}\right) \\
& \quad-Q_{-\omega}\left(z e^{-\pi i} ; N ; K_{2}, K_{1}\right) Q_{\omega}\left(z ; N ; K_{1}, K_{2}\right) \\
& =1+\sum_{l=1}^{\infty}(-)^{l N} \frac{K_{1}^{l} K_{2}^{l}}{z^{2 l N}} \sum_{j=0}^{2 l}(-)^{j} \tilde{G}_{\omega}^{(j)}(z ; N) \tilde{G}_{-\omega}^{(2 l-j)}\left(z e^{-\pi i} ; N\right),
\end{aligned}
$$

and, on combining this equation with (2.4), we derive the identity

$$
\begin{aligned}
P_{-\omega}\left(z e^{-\pi i} ;\right. & \left.N ; K_{2}, K_{1}\right) P_{\omega}\left(z ; N ; K_{1}, K_{2}\right) \\
& -Q_{-\omega}\left(z e^{-\pi i} ; N ; K_{2}, K_{1}\right) Q_{\omega}\left(z ; N ; K_{1}, K_{2}\right)=1 .
\end{aligned}
$$

The transformation of the hyperasymptotic expansions in $\mathbf{I}$ into the expansions presented in the following sections is based mainly on the following lemma.

Lemma 2.1. Let $N, M, l$ be arbitrary positive integers, and let $n \in\{0,1, \ldots, M-1\}$. Then

$$
\begin{aligned}
& G_{\omega}^{(l)}(z ; N, \ldots, N, N+M-n) \\
& =\sum_{j=0}^{l-1}(-)^{j+l-1} \tilde{G}_{\omega}^{(j)}(z ; N) \sum_{k=0}^{M-n-2} g_{(-)^{j} \omega}^{(l-j)}(N ; k+1)\left[(-)^{l} z\right]^{M-n-k-2} \\
& \quad+\left[(-)^{l} z\right]^{M-n-1} \tilde{G}_{\omega}^{(l)}(z ; N)
\end{aligned}
$$

(As is customary, empty sums are interpreted as being zero.) 
To prove (2.11), we substitute into the definition

$$
\begin{aligned}
& G_{\omega}^{(l)}(z ; N, \ldots, N, N+M-n) \\
& \quad=\int_{0}^{\infty} \cdots \int_{0}^{\infty} \frac{e^{-t-t_{1}-\cdots-t_{l-1}} t^{N+\omega} t_{1}^{N-\omega} \cdots t_{l-2}^{N+(-)^{l-2} \omega} t_{l-1}^{N+(-)^{l-1} \omega+M-n-1}}{(t+z)\left(t_{1}+t\right)\left(t_{2}+t_{1}\right) \cdots\left(t_{l-1}+t_{l-2}\right)} d t_{l-1} \cdots d t_{1} d t,
\end{aligned}
$$

by means of the identity

$$
\begin{aligned}
t_{l-1}^{M-n-1}=[ & \left.(-)^{l-1}(t+z)+(-)^{l}\left(t_{1}+t\right)+\sum_{j=2}^{l-1}(-)^{j+l-1}\left(t_{j}+t_{j-1}\right)\right] \\
& \times\left[\sum_{k=0}^{M-n-2} t_{l-1}^{k}\left[(-)^{l} z\right]^{M-n-k-2}\right]+\left[(-)^{l} z\right]^{M-n-1} .
\end{aligned}
$$

\section{Main results}

Theorem 3.1. For $p=0,1, \ldots$, define

$$
\begin{aligned}
T_{1}^{(p)}(z ; N, M)=\sum_{j=0}^{p}(-)^{j+\frac{1}{2} j(j-1+2 p) N} \frac{K_{1}^{\frac{1}{2}\left(j+\nu_{j}\right)} K_{2}^{\frac{1}{2}\left(j-\nu_{j}\right)}}{z^{p N}} \\
\quad \times \tilde{G}_{\omega}^{(j)}(z ; N) \sum_{s=0}^{M-1} \frac{a_{s, 1+\nu_{j}}^{p-j}(N)}{\left((-)^{j} z\right)^{s}}
\end{aligned}
$$

and

$$
\begin{aligned}
T_{2}^{(p)}(z ; N, M)=\sum_{j=0}^{p}(-)^{j+\frac{1}{2} j(j-1+2 p) N} \frac{K_{1}^{\frac{1}{2}\left(j-\nu_{j}\right)} K_{2}^{\frac{1}{2}\left(j+\nu_{j}\right)}}{z^{p N}} \\
\quad \times \tilde{G}_{-\omega}^{(j)}(z ; N) \sum_{s=0}^{M-1} \frac{a_{s, 2-\nu_{j}}^{p-j}(N)}{\left((-)^{j} z\right)^{s}}
\end{aligned}
$$

where $M$ and $N$ are arbitrary nonnegative integers. Then

$$
\begin{aligned}
& u_{1}(z)=\sum_{p=0}^{l} T_{1}^{(p)}(z ; N, N)+R_{1}^{(l)}(z ;(l+1) N, l N, \ldots, N) \\
& u_{2}(z)=\sum_{p=0}^{l} T_{2}^{(p)}(z ; N, N)+R_{2}^{(l)}(z ;(l+1) N, l N, \ldots, N) .
\end{aligned}
$$

Here the coefficients $a_{s, 1}^{m}(N)$ and $a_{s, 2}^{m}(N)$ are defined by $a_{s, 1}^{0}(N)=a_{s, 1}, a_{s, 2}^{0}(N)=a_{s, 2}$, 
and for $m=1,2, \ldots$,

$$
\begin{aligned}
& a_{s, 1}^{m}(N)=a_{N+s, 1}^{m-1}(N) \\
& \quad-(-)^{s m-\frac{1}{2} m(m+1) N} K_{1}^{\frac{1}{2}\left(m+\nu_{m}\right)} K_{2}^{\frac{1}{2}\left(m-\nu_{m}\right)} \sum_{n=0}^{s-1} a_{n, 1+\nu_{m}} g_{\omega}^{(m)}(N ; s-n) \\
& a_{s, 2}^{m}(N)=a_{N+s, 2}^{m-1}(N) \\
& \quad-(-)^{s m-\frac{1}{2} m(m+1) N} K_{1}^{\frac{1}{2}\left(m-\nu_{m}\right)} K_{2}^{\frac{1}{2}\left(m+\nu_{m}\right)} \sum_{n=0}^{s-1} a_{n, 2-\nu_{m}} g_{-\omega}^{(m)}(N ; s-n)
\end{aligned}
$$

Furthermore, as $N \rightarrow \infty,{ }^{1}$

$$
\begin{aligned}
& a_{s, 1}^{m}(N)=\left\{\prod_{j=0}^{m-1} \Gamma\left(N+(-)^{j} \Re \omega\right)\right\} \Gamma\left(s_{+}^{m}\right) \mathcal{O}_{m}(1), \\
& a_{s, 2}^{m}(N)=\left\{\prod_{j=0}^{m-1} \Gamma\left(N-(-)^{j} \Re \omega\right)\right\} \Gamma\left(s_{-}^{m}\right) \mathcal{O}_{m}(1),
\end{aligned}
$$

uniformly for $s=0,1, \ldots, N-1$, where $s_{ \pm}^{m}=\max \left(s \pm(-)^{m} \Re \omega, 1\right)$.

The expansions $(3.2 \mathrm{a}, \mathrm{b})$ are rearrangements of the expansions $(7.5 \mathrm{a}, \mathrm{b})$, respectively, of $\mathbf{I}$, and the estimates of the remainder terms supplied by Theorem 7.2 of $\mathbf{I}$ continue to apply. Thus, we have

$$
\begin{aligned}
& R_{1}^{(l)}(z ;(l+1) N, l N, \ldots, N)=\mathcal{O}\left(e^{-(l+1)|z|} z^{-\frac{1}{2} l+\nu_{l+1} \Re \omega}\right), \\
& R_{2}^{(l)}(z ;(l+1) N, l N, \ldots, N)=\mathcal{O}\left(e^{-(l+1)|z|} z^{-\frac{1}{2} l-\nu_{l+1} \Re \omega}\right),
\end{aligned}
$$

as $z \rightarrow \infty$ in $|\mathrm{ph} z| \leqslant \pi$.

\section{Theorem 3.2.}

$$
\begin{aligned}
& u_{1}(z)=P_{\omega}\left(z ; N ; K_{1}, K_{2}\right) A(z ; N)-Q_{\omega}\left(z ; N ; K_{1}, K_{2}\right) B(-z ; N), \\
& u_{2}(z)=P_{-\omega}\left(z ; N ; K_{2}, K_{1}\right) B(z ; N)-Q_{-\omega}\left(z ; N ; K_{2}, K_{1}\right) A(-z ; N),
\end{aligned}
$$

where the functions $A(z ; N)$ and $B(z ; N)$ are analytic in a deleted neighborhood of $z=\infty$. Furthermore, if $N$ is an integer such that $N=|z|+\mathcal{O}(1)$ as $|z| \rightarrow \infty$, then for each nonnegative integer $l$,

$$
\begin{aligned}
& A(z ; N)=\sum_{m=0}^{l} z^{-m N} \sum_{s=0}^{N-1} \frac{a_{s, 1}^{m}(N)}{z^{s}}+\mathcal{O}\left(e^{-(l+1)|z|} z^{-\frac{1}{2} l+\nu_{l+1} \Re \omega}\right), \\
& B(z ; N)=\sum_{m=0}^{l} z^{-m N} \sum_{s=0}^{N-1} \frac{a_{s, 2}^{m}(N)}{z^{s}}+\mathcal{O}\left(e^{-(l+1)|z|} z^{-\frac{1}{2} l-\nu_{l+1} \Re \omega}\right),
\end{aligned}
$$

as $z \rightarrow \infty$, uniformly with respect to unbounded $\mathrm{ph} z$.

\footnotetext{
${ }^{1}$ The $m$ in $\mathcal{O}_{m}$ indicates that the $\mathcal{O}$ term may depend on $m$.
} 
Owing to the extreme difficulty of computing the hyperconnection functions $P_{\omega}\left(z ; N ; K_{1}, K_{2}\right)$ and $Q_{\omega}\left(z ; N ; K_{1}, K_{2}\right)$, Theorem 3.2 is in the nature of an existence result. A more practical form is obtained on using (3.7) and truncating the expansions (2.7), as follows.

Theorem 3.3. Let $l$ be an arbitrary nonnegative integer, and $N=|z|+\mathcal{O}(1)$. Then as $z \rightarrow \infty$ in $|\mathrm{ph} z| \leqslant \pi$,

$$
\begin{aligned}
u_{1}(z)=[ & \left.\sum_{j=0}^{\left\lfloor\frac{1}{2} l\right\rfloor}(-)^{j N} \frac{K_{1}^{j} K_{2}^{j}}{z^{2 j N}} \tilde{G}_{\omega}^{(2 j)}(z ; N)\right]\left[\sum_{m=0}^{l} \frac{1}{z^{m N}} \sum_{s=0}^{N-1} \frac{a_{s, 1}^{m}(N)}{z^{s}}\right] \\
& -\left[\sum_{j=0}^{\left\lfloor\frac{1}{2} l-\frac{1}{2}\right\rfloor}(-)^{(j+1) N} \frac{K_{1}^{j+1} K_{2}^{j}}{z^{(2 j+1) N}} \tilde{G}_{\omega}^{(2 j+1)}(z ; N)\right]\left[\sum_{m=0}^{l} \frac{1}{(-z)^{m N}} \sum_{s=0}^{N-1} \frac{a_{s, 2}^{m}(N)}{(-z)^{s}}\right] \\
& +\mathcal{O}\left(e^{-(l+1)|z|} z^{-\frac{1}{2} l+\nu_{l+1} \Re \omega}\right),
\end{aligned}
$$

and

$$
\begin{aligned}
u_{2}(z)=[ & \left.\sum_{j=0}^{\left\lfloor\frac{1}{2} l\right\rfloor}(-)^{j N} \frac{K_{1}^{j} K_{2}^{j}}{z^{2 j N}} \tilde{G}_{-\omega}^{(2 j)}(z ; N)\right]\left[\sum_{m=0}^{l} \frac{1}{z^{m N}} \sum_{s=0}^{N-1} \frac{a_{s, 2}^{m}(N)}{z^{s}}\right] \\
& -\left[\sum_{j=0}^{\left\lfloor\frac{1}{2} l-\frac{1}{2}\right\rfloor}(-)^{(j+1) N} \frac{K_{1}^{j} K_{2}^{j+1}}{z^{(2 j+1) N}} \tilde{G}_{-\omega}^{(2 j+1)}(z ; N)\right]\left[\sum_{m=0}^{l} \frac{1}{(-z)^{m N}} \sum_{s=0}^{N-1} \frac{a_{s, 1}^{m}(N)}{(-z)^{s}}\right] \\
& +\mathcal{O}\left(e^{-(l+1)|z|} z^{-\frac{1}{2} l-\nu_{l+1} \Re \omega}\right),
\end{aligned}
$$

where $\lfloor p\rfloor$ is the integer $q$ such that $q \leqslant p<q+1$.

Theorems 3.1, 3.2, and 3.3 are proved in the following three sections.

\section{Proof of Theorem 3.1 for level one}

From $\mathbf{I}(3.12 \mathrm{a})$ and $\mathbf{I}(5.3)$ we obtain

$$
\begin{aligned}
R_{1}^{(0)}(z ; N)-R_{1}^{(1)}(z ; N+M, M) \\
=\sum_{s=N}^{N+M-1} \frac{a_{s, 1}}{z^{s}}+(-)^{N+M} \frac{K_{1}}{z^{N+M-1}} \sum_{n=0}^{M-1} a_{n, 2} G_{\omega}^{(1)}(z ; N+M-n) \\
=\sum_{s=N}^{N+M-1} \frac{a_{s, 1}}{z^{s}}-(-)^{N} \frac{K_{1}}{z^{N}} \sum_{n=0}^{M-2} \sum_{k=0}^{M-n-2} \frac{a_{n, 2} \Gamma(N+k+1+\omega)}{(-z)^{n+k+1}} \\
\quad-(-)^{N} \frac{K_{1}}{z^{N}} \tilde{G}_{\omega}^{(1)}(z ; N) \sum_{n=0}^{M-1} \frac{a_{n, 2}}{(-z)^{n}},
\end{aligned}
$$


where we have used Lemma 2.1, with $l=1$, and (2.6a). We can rewrite this expansion in the form

$$
\begin{aligned}
R_{1}^{(0)}(z ; N) & -R_{1}^{(1)}(z ; N+M, M) \\
& =z^{-N} \sum_{s=0}^{M-1} \frac{a_{s, 1}^{1}(N)}{z^{s}}-(-)^{N} \frac{K_{1}}{z^{N}} \tilde{G}_{\omega}^{(1)}(z ; N) \sum_{s=0}^{M-1} \frac{a_{s, 2}}{(-z)^{s}} \\
& =T_{1}^{(1)}(z ; N, M) .
\end{aligned}
$$

Similarly, we find that

$$
\begin{aligned}
& R_{2}^{(0)}(z ; N)-R_{2}^{(1)}(z ; N+M, M) \\
& \quad=z^{-N} \sum_{s=0}^{M-1} \frac{a_{s, 2}^{1}(N)}{z^{s}}-(-)^{N} \frac{K_{2}}{z^{N}} \tilde{G}_{-\omega}^{(1)}(z ; N) \sum_{s=0}^{M-1} \frac{a_{s, 1}}{(-z)^{s}} \\
& \quad=T_{2}^{(1)}(z ; N, M),
\end{aligned}
$$

where the new coefficients are defined as in (3.3). Notice that $a_{s, 1}^{1}(N)$ and $a_{s, 2}^{1}(N)$ are the remainders of asymptotic expansions $\mathbf{I}(3.17 \mathrm{a})$ and $\mathbf{I}(3.17 \mathrm{~b})$, respectively, after $s$ terms.

On combining (4.2) with the equations

$$
\begin{aligned}
& T_{1}^{(0)}(z ; N, M)=\sum_{s=0}^{M-1} \frac{a_{s, 1}}{z^{s}}=u_{1}(z)-R_{1}^{(0)}(z ; M) \\
& T_{2}^{(0)}(z ; N, M)=\sum_{s=0}^{M-1} \frac{a_{s, 2}}{z^{s}}=u_{2}(z)-R_{2}^{(0)}(z ; M)
\end{aligned}
$$

and taking $M=N$, we arrive at (3.2) in the case $l=1$.

When we substitute into $\mathbf{I}(3.11 \mathrm{a})$ by means of $\mathbf{I}(3.13)$, we obtain the integral representation

$$
a_{s, 1}=(-)^{s} K_{1} \int_{\rho}^{\infty} e^{-t} t^{s-1+\omega} u_{2}(t) d t+\frac{1}{2 \pi i} \int_{\rho e^{-\pi i}}^{\rho e^{\pi i}} t^{s-1} u_{1}(t) d t
$$

With the aid of this result and (3.3a) with $m=1$, we obtain

$$
\begin{aligned}
a_{s, 1}^{1}(N)=(-)^{N+s} K_{1} \int_{\rho}^{\infty} e^{-t} t^{N+s-1+\omega} u_{2}(t) d t+\frac{1}{2 \pi i} \int_{\rho e^{-\pi i}}^{\rho e^{\pi i}} t^{N+s-1} u_{1}(t) d t \\
\quad-(-)^{N+s} K_{1} \int_{0}^{\infty} e^{-t} t^{N+s-1+\omega} \sum_{n=0}^{s-1} \frac{a_{n, 2}}{t^{n}} d t \\
=(-)^{N+s} K_{1} \int_{\rho}^{\infty} e^{-t} t^{N+s-1+\omega} R_{2}^{(0)}(t ; s) d t+b_{s, 1}(N, \rho),
\end{aligned}
$$


where

$$
\begin{aligned}
b_{s, 1}(N, \rho)= & \frac{1}{2 \pi i} \int_{\rho e^{-\pi i}}^{\rho e^{\pi i}} t^{N+s-1} u_{1}(t) d t \\
& \quad-(-)^{N+s} K_{1} \sum_{n=0}^{s-1} a_{n, 2} \int_{0}^{\rho} e^{-t} t^{N+s-1+\omega-n} d t .
\end{aligned}
$$

We use $\mathbf{I}(3.8 \mathrm{a})$ and $\mathbf{I}(3.17 \mathrm{~b})$ in (4.5), and we find that

$$
b_{s, 1}(N, \rho)=\rho^{N+s} \mathcal{O}(1)+\Gamma\left(s_{+}^{1}\right) \rho_{1}^{N} \mathcal{O}(1)=\Gamma\left(s_{+}^{1}\right) \rho_{1}^{N} \mathcal{O}(1),
$$

where again $s_{ \pm}^{1}=\max (s \mp \Re \omega, 1)$ and $\rho_{1}=\max (\rho, 1)$. This estimate of $b_{s, 1}(N, \rho)$ is for $N \rightarrow \infty$, and it is uniform with respect to $s \geqslant 0$. From $\mathbf{I}(3.14 \mathrm{~b})$, with $\rho$ replaced by an arbitrary constant $\rho^{\prime}$ such that $a<\rho^{\prime}<\rho$, we see that $t^{s} R_{2}^{(0)}(t ; s)=\Gamma\left(s_{+}^{1}\right) \mathcal{O}(1)$, uniformly with respect to $t \geqslant \rho$ and $s \geqslant 0$. When we use this estimate in the integral in the final line of (4.4), we obtain

$$
(-)^{N+s} K_{1} \int_{\rho}^{\infty} e^{-t} t^{N+s-1+\omega} R_{2}^{(0)}(t ; s) d t=\Gamma(N+\Re \omega) \Gamma\left(s_{+}^{1}\right) \mathcal{O}(1)
$$

as $N \rightarrow \infty$, uniformly with respect to $s \geqslant 0$. On substituting into (4.4) by means of the last two results and using a similar analysis for $a_{s, 2}^{1}(N)$, we find that (3.4) is valid for $m=1$. Thus the behavior of the terms of the first sum in (4.2a) is (approximately) as follows. The first term is of the same order of magnitude as the left-hand side; the following terms decrease until $s=N$, thereafter they increase. The terms in the second sum in (4.2a) exhibit similar behavior, as do those in the sums in (4.2b).

\section{Proof of Theorem 3.1 for level two}

From I (6.2) we obtain

$$
\begin{aligned}
R_{1}^{(1)}(z ; 2 N, & N)-R_{1}^{(2)}(z ; 2 N+M, N+M, M) \\
=\{ & \left.R_{1}^{(1)}(z ; 2 N, N)-R_{1}^{(0)}(z ; N)\right\} \\
& \quad+\left\{R_{1}^{(0)}(z ; N)-R_{1}^{(1)}(z ; 2 N+M, N+M)\right\} \\
& +(-)^{N} \frac{K_{1} K_{2}}{z^{2 N+M-1}} \sum_{n=0}^{M-1} a_{n, 1} G_{\omega}^{(2)}(z ; N, N+M-n) .
\end{aligned}
$$

Again we use Lemma 2.1 and the first line of (4.2a). We find that

$$
\begin{aligned}
& R_{1}^{(1)}(z ; 2 N, N)-R_{1}^{(2)}(z ; 2 N+M, N+M, M) \\
& =z^{-2 N} \sum_{s=0}^{M-1} \frac{a_{s, 1}^{2}(N)}{z^{s}}-\frac{K_{1}}{z^{2 N}} \tilde{G}_{\omega}^{(1)}(z ; N) \sum_{s=0}^{M-1} \frac{a_{s, 2}^{1}(N)}{(-z)^{s}} \\
& \quad+(-)^{N} \frac{K_{1} K_{2}}{z^{2 N}} \tilde{G}_{\omega}^{(2)}(z ; N) \sum_{s=0}^{M-1} \frac{a_{s, 1}}{z^{s}} \\
& =T_{1}^{(2)}(z ; N, M),
\end{aligned}
$$


where the coefficients $a_{s, 1}^{2}(N)$ are defined by (3.3a) with $m=2$. Similarly,

$$
\begin{aligned}
& R_{2}^{(1)}(z ; 2 N, N)-R_{2}^{(2)}(z ; 2 N+M, N+M, M) \\
& =z^{-2 N} \sum_{s=0}^{M-1} \frac{a_{s, 2}^{2}(N)}{z^{s}}-\frac{K_{2}}{z^{2 N}} \tilde{G}_{-\omega}^{(1)}(z ; N) \sum_{s=0}^{M-1} \frac{a_{s, 1}^{1}(N)}{(-z)^{s}} \\
& \quad+(-)^{N} \frac{K_{1} K_{2}}{z^{2 N}} \tilde{G}_{-\omega}^{(2)}(z ; N) \sum_{s=0}^{M-1} \frac{a_{s, 2}}{z^{s}} \\
& =T_{2}^{(2)}(z ; N, M) .
\end{aligned}
$$

The coefficients can be estimated in a similar way to that used for $a_{s, 1}^{1}(N)$ and $a_{s, 2}^{1}(N)$ in $\S 4$, and we obtain (3.4) with $m=2$. It follows that all of the sums in (5.2) have optimal behavior for divergent series: the first terms are of the same magnitude as the left-hand side, the terms then decrease in magnitude for $0 \leqslant s<N$, and increase in magnitude for $s>N$.

\section{Higher levels: completion of the proofs of Theorems 3.1, 3.2, and 3.3}

The analysis used in $\S 5$ may be continued to levels three and higher, and Theorem 3.1 follows straightforwardly by induction.

We next prove Theorem 3.3. From (3.4) and I, Lemma 2.1, we see that if $j$ and $m$ are nonnegative integers such that $j+m \geqslant l+1$, then

$$
\frac{\tilde{G}_{\omega}^{(j)}(z ; N)}{z^{(m+j) N}} \sum_{s=0}^{N-1} \frac{a_{s, 1+\nu_{j}}^{m}(N)}{\left((-)^{j} z\right)^{s}}=\mathcal{O}\left(e^{-(l+1)|z|} z^{-\frac{1}{2} l+\nu_{l+1} \Re \omega}\right)
$$

as $z \rightarrow \infty$ in $|\mathrm{ph} z| \leqslant \pi$. When we substitute into (3.2a) by means of (3.1a) and (3.5a) and apply (6.1) with $m=p-j$, we obtain

$$
\begin{aligned}
& u_{1}(z)=\sum_{p=0}^{l} \sum_{j=0}^{p}(-)^{j+\frac{1}{2} j(j-1+2 p) N} \frac{K_{1}^{\frac{1}{2}\left(j+\nu_{j}\right)} K_{2}^{\frac{1}{2}\left(j-\nu_{j}\right)}}{z^{p N}} \tilde{G}_{\omega}^{(j)}(z ; N) \sum_{s=0}^{N-1} \frac{a_{s, 1+\nu_{j}}^{p-j}(N)}{\left((-)^{j} z\right)^{s}}+\mathcal{O}^{*} \\
& =\sum_{j=0}^{l} \sum_{m=0}^{l-j}(-)^{j+j m N+\frac{1}{2} j(j+1) N} \frac{K_{1}^{\frac{1}{2}\left(j+\nu_{j}\right)} K_{2}^{\frac{1}{2}\left(j-\nu_{j}\right)}}{z^{(m+j) N}} \tilde{G}_{\omega}^{(j)}(z ; N) \sum_{s=0}^{N-1} \frac{a_{s, 1+\nu_{j}}^{m}(N)}{\left((-)^{j} z\right)^{s}}+\mathcal{O}^{*} \\
& =\sum_{j=0}^{l}(-)^{j+\frac{1}{2} j(j+1) N} \frac{K_{1}^{\frac{1}{2}\left(j+\nu_{j}\right)} K_{2}^{\frac{1}{2}\left(j-\nu_{j}\right)}}{z^{j N}} \tilde{G}_{\omega}^{(j)}(z ; N) \sum_{m=0}^{l}\left((-)^{j} z\right)^{-m N} \sum_{s=0}^{N-1} \frac{a_{s .1+\nu_{j}}^{m}(N)}{\left((-)^{j} z\right)^{s}}+\mathcal{O}^{*},
\end{aligned}
$$

where, for brevity, we have denoted the $\mathcal{O}$-term in (6.1) by $\mathcal{O}^{*}$. On splitting the $j$ sum in the last equation into even and odd terms, we arrive at (3.8a). Similarly for (3.8b).

To prove Theorem 3.2, we first observe from (2.9) and $\mathbf{I}(3.9)$ that the functions $A(z ; N)$ and $B(z ; N)$ defined by

$$
\begin{gathered}
A(z ; N)=P_{-\omega}\left(z e^{-\pi i} ; N ; K_{2}, K_{1}\right) u_{1}(z)+Q_{\omega}\left(z ; N ; K_{1}, K_{2}\right) u_{2}\left(z e^{-\pi i}\right), \\
B(z ; N)=P_{\omega}\left(z e^{\pi i} ; N ; K_{1}, K_{2}\right) u_{2}(z)+Q_{-\omega}\left(z ; N ; K_{2}, K_{1}\right) u_{1}\left(z e^{\pi i}\right)
\end{gathered}
$$


satisfy $A\left(z e^{2 \pi i} ; N\right)=A(z ; N)$ and $B\left(z e^{2 \pi i} ; N\right)=B(z ; N)$. They are therefore analytic in a deleted neighborhood of $z=\infty$. On combining (2.10) with (6.2) we obtain equations (3.6).

It remains to prove (3.7). From (2.8), we see that we can rewrite the expansions (3.8) in the form

$$
\begin{aligned}
u_{1}(z)= & P_{\omega}\left(z ; N ; K_{1}, K_{2}\right) \sum_{m=0}^{l} z^{-m N} \sum_{s=0}^{N-1} \frac{a_{s, 1}^{m}(N)}{z^{s}} \\
& \quad-Q_{\omega}\left(z ; N ; K_{1}, K_{2}\right) \sum_{m=0}^{l}(-z)^{-m N} \sum_{s=0}^{N-1} \frac{a_{s, 2}^{m}(N)}{(-z)^{s}} \\
& +\mathcal{O}\left(e^{-(l+1)|z|} z^{-\frac{1}{2} l+\nu_{l+1} \Re \omega}\right), \\
u_{2}(z)= & P_{-\omega}\left(z ; N ; K_{2}, K_{1}\right) \sum_{m=0}^{l} z^{-m N} \sum_{s=0}^{N-1} \frac{a_{s, 2}^{m}(N)}{z^{s}} \\
& -Q_{-\omega}\left(z ; N ; K_{2}, K_{1}\right) \sum_{m=0}^{l}(-z)^{-m N} \sum_{s=0}^{N-1} \frac{a_{s, 1}^{m}(N)}{(-z)^{s}} \\
& +\mathcal{O}\left(e^{-(l+1)|z|} z^{-\frac{1}{2} l-\nu_{l+1} \Re \omega}\right),
\end{aligned}
$$

as $z \rightarrow \infty$ in $|\mathrm{ph} z| \leqslant \pi$. When $0 \leqslant \mathrm{ph} z \leqslant \pi$, we may substitute (6.3) into (6.2) and apply (2.8) and (2.10). We find that as $z \rightarrow \infty$

$$
\begin{aligned}
A(z ; N)= & \sum_{m=0}^{l} z^{-m N} \sum_{s=0}^{N-1} \frac{a_{s, 1}^{m}(N)}{z^{s}} \\
& \quad+P_{-\omega}\left(z e^{-\pi i} ; N ; K_{2}, K_{1}\right) \mathcal{O}\left(e^{-(l+1)|z|} z^{-\frac{1}{2} l+\nu_{l+1} \Re \omega}\right) \\
& \quad+Q_{\omega}\left(z ; N ; K_{1}, K_{2}\right) \mathcal{O}\left(e^{-(l+1)|z|} z^{-\frac{1}{2} l-\nu_{l+1} \Re \omega}\right) \\
= & \sum_{m=0}^{l} z^{-m N} \sum_{s=0}^{N-1} \frac{a_{s, 1}^{m}(N)}{z^{s}}+\mathcal{O}\left(e^{-(l+1)|z|} z^{-\frac{1}{2} l+\nu_{l+1} \Re \omega}\right) .
\end{aligned}
$$

Alternatively, if $-\pi \leqslant \operatorname{ph} z \leqslant 0$, then with the aid of (2.9), (6.3), and $\mathbf{I}(3.9)$, we can show that $(6.3 \mathrm{~b})$ also applies when $z$ is replaced by $z e^{-\pi i}$, provided that the $\mathcal{O}$-term is changed by a factor $e^{-z} z^{-\omega}$. Hence (6.4) continues to apply. Since $A(z ; N)$ is analytic, we have established (3.7a) uniformly for unrestricted ph $z$. Similarly for $(3.7 \mathrm{~b})$.

Remark. It is not clear whether the expansions (3.7) converge as $l \rightarrow \infty$. This would appear to depend on how the estimates $\mathcal{O}_{m}(1)$ in (3.4) grow with $m$.

\section{Comparison of the various hyperasymptotic expansions}

We now have five optimized expansions of hyperasymptotic type for the basic functions $u_{1}(z)$ and $u_{2}(z)$, given by Theorems 7.2 and 8.1 of $\mathbf{I}$, and by Theorems 3.1, 3.2, and 3.3 of the present paper.

All five theorems include the sector $|\mathrm{ph} z| \leqslant \pi$ in their region of validity. This suffices for practical applications, since we can employ the connection formulas I (3.9) elsewhere. However, only Theorem 3.2 applies directly to unrestricted values of ph $z$. 
As we have already noted in $\S 3$, Theorem 3.2 is in the nature of an existence theorem. It shows, for example, that with the normalization $\lambda_{2}-\lambda_{1}=1$ the qualitative behavior of the basic functions in the neighborhoods of the Stokes lines ph $z= \pm \pi, \pm 2 \pi, \ldots$, depends only on the connection coefficients $K_{1}, K_{2}$ and the parameter $\omega \equiv \mu_{2}-\mu_{1}$.

From the standpoint of applications, Theorem 7.2 and 8.1 of $\mathbf{I}$ have already been compared in I. We now compare Theorems 3.1 and 3.3 with Theorem 7.2 of $\mathbf{I}$. One feature of the latter that differs from the usual type of generalized asymptotic expansion is that, as we proceed from one level of approximation to the next, extra terms have to be inserted at all preceding levels. A second feature, and one which is a major drawback, is that, at levels $3,4, \ldots$, the hyperasymptotic expansions rapidly become unstable owing to numerical cancellation in the summation process. In contrast, once the coefficients $a_{s, 1}^{m}(N)$ and $a_{s, 2}^{m}(N)$ in Theorems 3.1 and 3.3 have been calculated, the expansions (3.2) and (3.8) do not exhibit either of these features. However, these features are not altogether absent; they reappear when we consider the calculation of $a_{s, 1}^{m}(N)$ and $a_{s, 2}^{m}(N)$ by means of formulas (3.3). On proceeding from one level to the next, we have to compute $N$ new coefficients at each of the preceding levels. This also means that $a_{s, 1}^{l}(N)$ and $a_{s, 2}^{l}(N)$ are, in effect, linear combinations of the original coefficients $a_{s, 1}$ and $a_{s, 2}$ for $s=0,1, \ldots,(l+1) N-1$. These are precisely the coefficients that appear explicitly in the hyperasymptotic expansions supplied by I, Theorem 7.2. In consequence, the same kind of numerical instability will occur on summing the right-hand sides of equations (3.3).

For the foregoing reasons, there is no gain in using Theorems 3.1 and 3.3 in preference to Theorem 7.2 of $\mathbf{I}$ for isolated values of $z$. Indeed, there is a loss, since the expansions supplied by Theorems 3.1 and 3.3, namely (3.2), (3.3), and (3.8), are more complicated. However, Theorems 3.1 and 3.3 will gain if several values of $z$ are needed that differ by $\mathcal{O}(1)$ in magnitude. In this situation, the same value of $N$, and hence the same values of $a_{s, 1}^{m}(N)$ and $a_{s, 2}^{m}(N)$, can be used throughout. Theorems 3.1 and 3.3 would also gain, indeed gain overwhelmingly, if a numerically stable way of evaluating these coefficients could be found for all levels.

In comparing the expansions furnished by Theorems 3.1 and 3.3, we note that the estimates for the remainder terms are the same, hence the expansions should yield comparable accuracy in applications. Theorem 3.3 has the advantage that it entails less computation. The expansions (3.8) are in terms of double sums multiplied by single sums, whereas the expansions (3.2) are in effect triple sums; compare (3.1). However, Theorem 3.1 has the advantage that it supplies explicit integral representations for the remainder terms (see $\mathbf{I}(5.6)$ and $\mathbf{I}(6.3)$ ).

In summary, for isolated values of $z$, the weaker but numerically stable version of Theorem 7.2 of $\mathbf{I}$, namely Theorem 8.1 of $\mathbf{I}$, is to be preferred in applications. For a range of values of $z$ that differ by $\mathcal{O}(1)$ in magnitude, Theorems 3.1 and 3.3 may be advantageous, especially when the coefficients that are needed are calculable in a numerically stable manner.

Lastly, the problems of evaluating the multiple integrals $G_{ \pm \omega}^{(l)}$ that were discussed in the concluding section of $\mathbf{I}$ persist in the calculation of the multiple integrals $\tilde{G}_{ \pm \omega}^{(l)}$ and $g_{ \pm \omega}^{(l)}$. These problems will need to be resolved if the use of hyperasymptotic expansions at higher levels is to become a practicable tool. 


\section{Example}

We take the same example as in $\mathbf{I}$, namely the differential equation

$$
\frac{d^{2} w}{d z^{2}}+\left(\frac{1}{z}-1\right) \frac{d w}{d z}-\frac{1}{2 z} w=0
$$

satisfied by $e^{\frac{1}{2} z} K_{0}\left(\frac{1}{2} z\right)$ and $e^{\frac{1}{2} z} K_{0}\left(\frac{1}{2} z e^{-\pi i}\right)$, where $K_{0}$ is the modified Bessel function. We have $\lambda_{1}=0, \lambda_{2}=1, \mu_{1}=\mu_{2}=-\frac{1}{2}$, and $\omega=0$. The two basic functions and the coefficients in $\mathbf{I}(3.7)$ to $\mathbf{I}(3.9)$ are given by

$$
u_{1}(z)=u_{2}(z)=\pi^{-\frac{1}{2}} z^{\frac{1}{2}} e^{\frac{1}{2} z} K_{0}\left(\frac{1}{2} z\right), \quad a_{s, 1}=a_{s, 2}=(-)^{s} \frac{1^{2} \cdot 3^{2} \cdot \ldots \cdot(2 s-1)^{2}}{4^{s} s !},
$$

and $K_{1}=K_{2}=\pi^{-1}$.

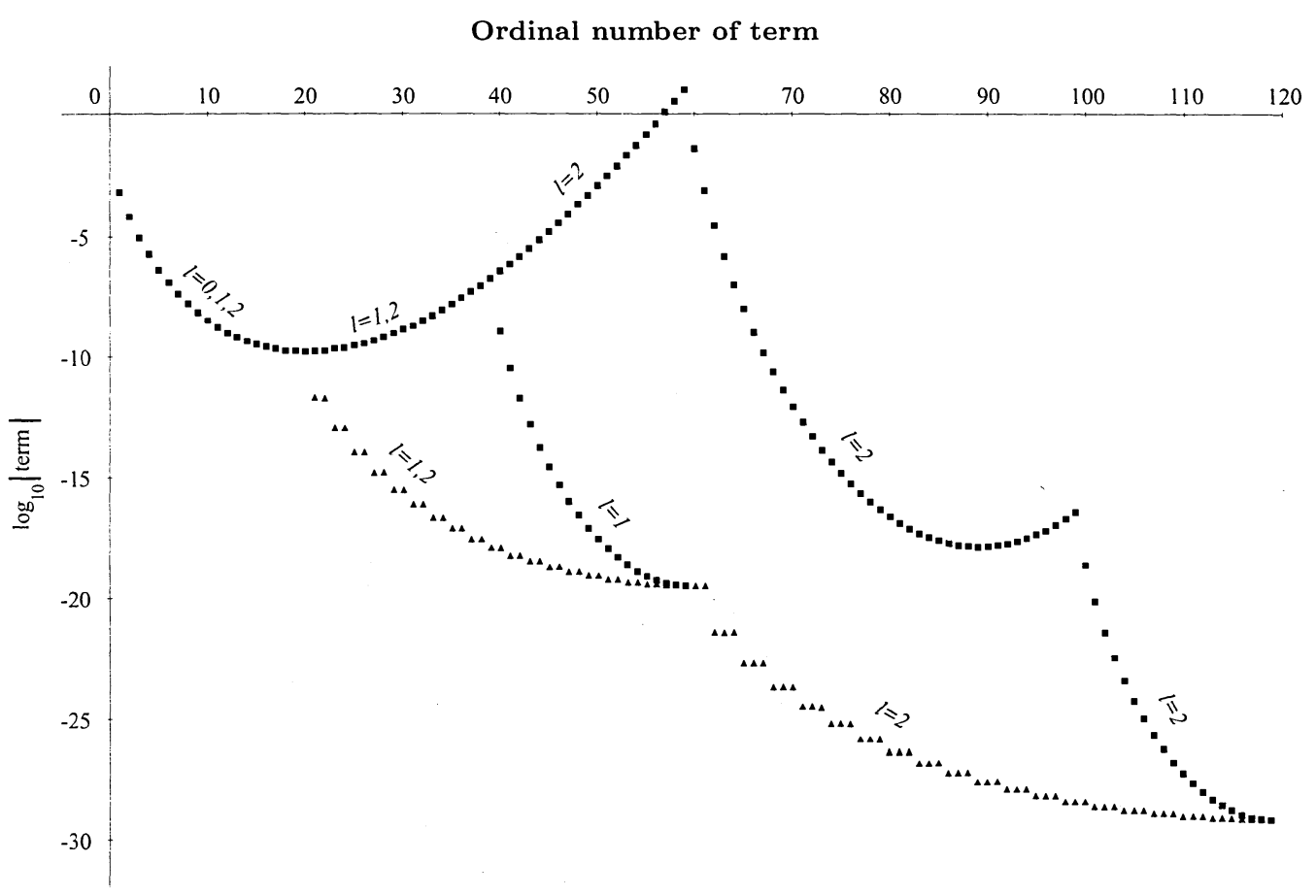

FiguRE 8.1. Magnitudes of the terms in hyperasymptotic expansions

(7.5) of $\mathbf{I}$ and (3.1) of the present paper.

Again we take $z=20$ and hence $N=20$. The squares in Figure 8.1 are the plot of the logarithm to base 10 of the absolute value of each term in the expansion $\mathbf{I}(7.5 \mathrm{a})$ against its ordinal number. For $l=0$, the expansion reduces to the original asymptotic expansion $\mathbf{I}(3.8 \mathrm{a})$, and it is terminated at (or very nearly at) the smallest term. For $l=1$, we take 40 terms of the original expansion, and then 20 terms of the first re-expansion. Again this re-expansion is terminated at its smallest term. And for $l=2$, we take 60 terms of the original expansion, 40 terms of the first re-expansion, and 20 terms of the second re-expansion, thereby terminating the second re-expansion 
at its smallest term. As in $\mathbf{I}$, we note that a few terms rise above unity in magnitude indicating some instability in the summation.

The curves indicated by the triangles plot the terms in the expansion (3.2a) for $l=1,2$. (For $l=0$, the curve is the same as that for $\mathbf{I}(7.5 \mathrm{a})$.) For $l=1$, we start with the $l=0$ expansion and then add (3.1a) with $p=1$ and $M=N=20$ for the first re-expansion. ${ }^{2}$ Due to the $j$-sums in (3.1a), the triangles appear pairwise. This first re-expansion is terminated at its smallest term. For $l=2$, we start with the $l=1$ expansion, and then add (3.1a) with $p=2$ and $M=N=20$ for the second re-expansion. Now the triangles appear in groups of three. Again, the second re-expansion is terminated at its smallest term.

We observe that at each level the optimal number of terms in the expansions (3.2a) and $\mathbf{I}(7.5 \mathrm{a})$ are the same and that the final terms in each expansion are of the same order of magnitude. We also observe that the curve of triangles indicates that the expansion (3.2a) is numerically stable and that at each level this expansion is terminated at an optimal stage.

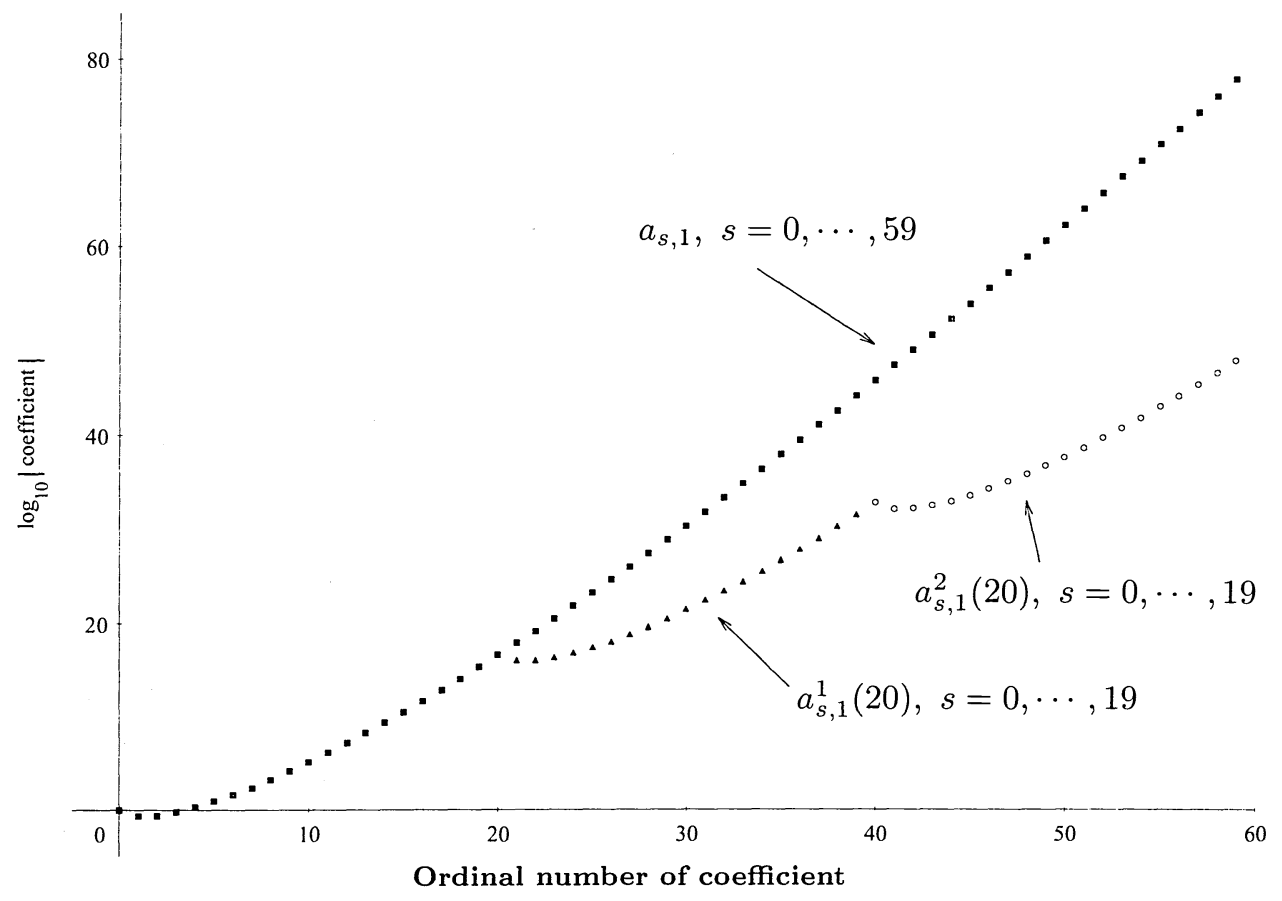

Figure 8.2. Magnitudes of the coefficients in the first three levels.

We illustrate also the numerical instability in the computation of the coefficients in (3.1) by means of (3.3). From (3.3a) and (2.6) we obtain

$$
a_{s, 1}^{1}(N)=a_{N+s, 1}-(-)^{N+s} K_{1} \sum_{n=0}^{s-1} a_{n, 2} \Gamma(N+s-n+\omega)
$$

\footnotetext{
${ }^{2}$ The reason that we take $M=N=20$ is that for general $M$ and $N$ the remainder is $R_{1}^{(1)}(z ; N+M, M)$ (see $\left.(4.2 a)\right)$. In $\mathbf{I}$, we showed that this remainder is minimal for $M=N \approx|z|$.
} 
and

$$
\begin{aligned}
a_{s, 1}^{2}(N) & =a_{2 N+s, 1}-(-)^{s} K_{1} \sum_{n=0}^{N+s-1} a_{n, 2} \Gamma(2 N+s-n+\omega) \\
& -(-)^{N} K_{1} K_{2} \sum_{n=0}^{s-1} a_{n, 1} \frac{\Gamma(N+1+\omega) \Gamma(N+s-n-\omega)}{2 N+s-n} .
\end{aligned}
$$

Figure 8.2 plots the logarithm to base 10 of the coefficients we have used in (3.2a). The discrepancy in the order of magnitudes of the $a_{s, 1}^{1}(20)$ (indicated by the triangles) from those of the $a_{20+s, 1}$ (indicated by the squares directly above) reveals the extent of the cancellation that takes place on evaluating the right-hand side of (8.1). The discrepancy is even greater for the $a_{s, 1}^{2}(20)$ and $a_{40+s, 1}$ (compare the circles with the squares directly above). Thus the cancellation associated with equation (8.2) is considerably more severe than that associated with (8.1).

\section{References}

1. A. B. Olde Daalhuis and F. W. J. Olver, Hyperasymptotic solutions of second-order linear differential equations I, Methods Appl. Anal. 2 (1995), 173-197.

Institute for Physical Science and Technology, University of Maryland, College Park, MARYLAND 20742, U.S.A.

E-mail: aod@olgao.umd.edu 